\title{
L'impact des attitudes et représentations des langues sur l'enseignement. Le cas du français en Algérie
}

\author{
Dr. Outaleb-Pellé, Aldjia \\ Département de français \\ Université de Tizi Ouzou \\ Laboratoire « Pratiques langagières » \\ a.outaleb_pelle@yahoo.fr
}

\section{Introduction}

La notion de « représentations » des sujets sur les langues est, de nos jours, de plus en plus présente en linguistique, notamment dans l'enseignement-apprentissage (Véronique 1990, Zarate 1993 ; Muller 1998). En effet, ces images, fortement stéréotypées, recèlent un pouvoir valorisant ou, a contrario, inhibant vis-à-vis de l'apprentissage et de la pratique des langues.

Dans cette perspective, nous nous posons ces questions :

- A quelle réalité renvoie la configuration linguistique en Algérie ?

- Quels statuts occupent les langues en présence?

- Quelles représentations se font les locuteurs des différentes langues?

- Quel est l'impact de ces représentations sur l'enseignement des langues ?

- Quelles stratégies adopter pour l'enseignement du FLE dans une communauté plurilingue ?

Notre article va s'articuler sur deux axes : une partie théorique et une partie pratique. Dans la première, nous évoquerons la question du plurilinguisme algérien en général ; tizi ouzien en particulier et du rôle et la place du français dans le système scolaire algérien. Dans la seconde partie, nous présenterons la méthodologie suivie, l'analyse et l'interprétation des données recueillies. Enfin, une sorte de synthèse permettra de reprendre les grands axes traités ainsi que l'impact sur la motivation à l'apprentissage du français.

\section{Coexistence et guerre des langues}

Le contexte étant un facteur important dans la formation des représentations il est nécessaire d'établir un état des lieux du cadre sociolinguistique algérien afin de cerner l'image qui s'offre à l'apprenant.

Comme le soutiennent les discours officiels qui consistent à nier l'existence d'autres langues minoritaires ou régionales, la société algérienne n'est pas une société bilingue encore moins monolingue.

Les études menées par Taleb-Ibrahimi, 1995 ; Morsly, 1988, exposent la richesse linguistique des Algériens et leur extrême aisance à utiliser des alternances codiques, un code mixing, qui mêlent l'arabe avec ses différentes variantes, le français avec ses normes endogènes, et le berbère avec ses différents dialectes comme étant des stratégies discursives et communicatives. En effet, une configuration linguistique quadridimensionnelle est composée fondamentalement de l'arabe algérien, la langue maternelle et parlée de la majorité, de l'arabe classique ou standard, pour l'usage de l'officialité, de la langue française pour l'enseignement scientifique, le savoir et du berbère, autre langue maternelle d'une autre partie de la population.

En Algérie, la politique linguistique, basée sur l'installation d'une seule langue, en rapport avec le nationalisme, revendique la valorisation de l'arabe classique à laquelle on accorde le statut de langue 
nationale et officielle. Du coup, cette langue marginalise les langues maternelles à un statut moindre (l'arabe dialectal et ses variétés et le berbère reconnu langue nationale en 2002).

Quant au français, l'Algérie n'a jamais tranché la question de son rôle et de sa place. Au fil du temps et des réformes scolaires, divers statuts lui ont été attribués tels ceux de "langue de spécialité ", « langue étrangère privilégiée ", " langue des sciences et techniques ». Et depuis quelques années, celui de " langue étrangère ». Ceci dit, c'est surtout grâce aux échanges communicationnels oraux et non pas uniquement par l'enseignement-apprentissage octroyé par l'institution scolaire que le français a pris place dans la vie quotidienne des Algériens.

Il ne nous est pas possible, dans le cadre de cet article, de dresser un tableau complet de la situation des langues. Ces indications suffisent pour nous permettre de poser brièvement les repères nécessaires aux statuts de ces langues. En outre, c'est précisément les rapports avec la langue française qu'il s'agit de mettre en exergue. Ce qui nous amène à nous demander :

- Quelle place le français occupe dans la société algérienne, en général, et à Tizi Ouzou, en particulier ;

- Quels rapports le français entretient avec les autres langues locales ;

- Quel impact il génère quant à son enseignement.

\section{Présentation générale de Tizi Ouzou : une ville plurilingue}

Située au centre nord de l'Algérie, à 100 kilomètres à l'est d'Alger, Tizi Ouzou se présente comme la capitale de la Grande Kabylie. Elle couvre une superficie de 10236 hectares et a une population avoisinant les 150088 habitants (2008).

La ville de Tizi Ouzou ne s'est pas retrouvée «plurielle » du jour au lendemain ; il a fallu la présence et le contact de peuples et de langues, et ce, pendant des siècles.

De toutes les colonisations subies par l'Algérie, deux ont reconfiguré sa situation linguistique : d'un côté, les mouvements d'islamisation ont fait que l'arabe s'est introduit et a pris au berbère son statut de langue de communication quotidienne ; de l'autre, avec l'invasion française, l'Algérie se retrouve dans un paysage linguistique nouveau marqué par la domination du français sur les langues locales.

Juste après l'indépendance, en 1962, la politique linguistique puissamment menée a abouti à l'émergence de l'arabe classique dans toutes les institutions. Cependant, pour les habitants de Tizi Ouzou, berbérophones, s'est prolongé le rapport avec le français bien intégré et conjuguant avec le kabyle et l'arabe tizi ouzéen (parler résultant d'un mélange fondé sur le lexique kabyle avec une morphologie arabe).

Formée par l'école française, la Kabylie présente ainsi un particularisme linguistique.

De grands écrivains, comme Jean et Taous Amrouche, Mouloud Feraoun, Mouloud Mammeri, ont produit une œuvre littéraire en langue française. De ce fait, ces auteurs ont du subir des critiques d'autres intellectuels qui leur ont reproché d'être des assimilés de la France.

Le français s'est enraciné dans la société kabyle et jusqu'à présent, les Kabyles sont de bons locuteurs francophones même s'ils ne maitrisent pas tous la langue écrite. Ils sont tous capables de mener une conversation en cette langue qui ne leur est pas du tout étrangère et qu'ils manient avec beaucoup d'aisance.

L'enseignement-apprentissage du français en Algérie tributaire de l'ambiguïté due à son statut n'accorde pas d'intérêt aux spécificités régionales très importantes car elles déterminent les différentes attitudes envers l'enseignement-apprentissage. Et s'agissant de la région de Tizi Ouzou, des particularités doivent être relevées : l’Histoire coloniale et sa situation géographique.

La région kabyle s'est vite « francisée » du fait de l'installation des Pères Blancs et des Sours Blanches, qui ont beaucoup aidé les populations à sortir de l'analphabétisme et de la misère. Malgré sa situation géographique (les hautes montagnes du Djurdjura à climat très rude) les villageois ont amélioré leurs 
conditions de vie en allant travailler en métropole comme ouvriers. C'est ainsi que des allers-retours incessants ont toujours été effectués entre la France et la Kabylie.

Notre étude s'interroge sur l'influence que peut induire cette particularité de la région sur les représentations qu'ont les apprenants de la France, de la langue française et de son enseignementapprentissage.

\section{Le français dans le système scolaire algérien}

L'institution scolaire est le lieu des apprentissages pour former un bon citoyen et le français «langue étrangère » est considérée en Algérie comme étant la langue de prestige et de la réussite professionnelle. Il est souligné dans les instructions officielles que «le français défini comme moyen d'ouverture sur le monde extérieur doit permettre à la fois l'accès à une documentation scientifique d'une part mais aussi le développement des échanges entre les civilisations et la compréhension mutuelle entre les peuples » (Ordonnance n`76/35 du 16 avril 1976 portant organisation de l'éducation et de la formation, reprise en 2006).

Assurer la communication en français dont l'apprenant doit maitriser le fonctionnement est le principal objectif annoncé dans les programmes scolaires «c'est avant tout apprendre à communiquer dans cette langue pour établir des échanges discursifs et interactifs » (Ordonnance du 16 avril 1976 reprise en 2006).

Les programmes scolaires se traduisent ainsi en termes de compétences générales à faire acquérir aux élèves, d'objectifs spécifiques et de contenus. Ils s'articulent autour de la compréhension de l'écrit, du fonctionnement de la langue et de la production écrite.

Les contenus des programmes proposent de travailler la langue en lien étroit avec les typologies textuelles. Quatre formes de discours sont privilégiées : le narratif, le descriptif, l'expositif, l'explicatif enfin, l'argumentatif avec toutes les combinaisons thématiques qui peuvent se présenter dans un texte. Une perspective du discours, retenue pour un ensemble spécifique du programme, permet d'étudier aussi bien la grammaire du discours, la grammaire de texte et la grammaire de la phrase.

L'apprentissage part donc des textes écrits d'auteurs qui font découvrir aux élèves, à l'aide de questions inductives, les notions inhérentes aux formes du discours retenu. Par exemple, les notions inhérentes au texte descriptif répondent à la caractérisation; dans cette perspective, le groupe nominal, l'adjectif, le complément du nom, la relative, l'expression de l'opposition et celle de la comparaison seront étudiés.

Chaque dossier finit par une production écrite suivie de sa correction. Ses objectifs sont clairement définis : l'élève doit être en mesure de produire un texte conforme aux normes du texte étudié auparavant. Autrement dit, il doit être capable de réaliser un écrit selon les divers types de discours tracés dans les programmes.

Le volume horaire imparti au français est le suivant :

- A l'école primaire : les enfants sont scolarisés dès l'âge de six ans et commencent à apprendre le français à partir de la $3 e$ année. De la $3 e$ à la 6e année, dernière classe du primaire, le volume horaire réservé à l'enseignement du français est de 4 heures par semaine.

- Après le cycle primaire, les élèves suivent une scolarité de quatre ans, au collège où, là encore, l'enseignement du français est assuré, à raison de 3 heures par semaine.

- Au lycée, les études durent également trois ans et les cours de français sont répartis à raison de 3 heures par semaine.

Arrivés en fin de cursus scolaire, classe de terminale, les élèves auront suivi pendant onze ans l'enseignement-apprentissage du français et ce, depuis la 3e année du primaire jusqu'en classe de terminale.

Jusqu'à présent, bien qu'avec la nouvelle réforme, les programmes accordent un enseignement du français, à partir de la 3e année de l'école primaire, ce qui pourrait être interprété comme une volonté politique de conforter l'apprentissage du français en l'introduisant juste après l'arabe standard, le français demeure officiellement une langue étrangère, une matière à enseigner, étudiée en elle-même et pour elle- 
même, c'est-à-dire qu'elle n'est pas au service de l'apprentissage d'autres matières ; et ne doit renfermer aucune dimension culturelle.

Bien que l'apprentissage de la langue française commence à partir de la 3e année du primaire le volume horaire, le faible coefficient, la mauvaise répartition horaire du français (généralement enseigné en fin d'après midi) ne permettent pas aux élèves d'acquérir une bonne maîtrise de la langue. En effet, à la fin du cursus scolaire, les élèves sont censés avoir un bagage linguistique en français leur permettant de poursuivre aisément des études universitaires. Mais, le constat est tout autre : ayant accumulé, avec le temps, beaucoup de lacunes, les apprenants éprouvent la plus grande peine à s'exprimer correctement en français (Outaleb-Pellé, 2010).

\section{Méthodologie de recherche choisie}

Pour réaliser le volet pratique de cette recherche, qui est une analyse des comportements d'un échantillon d'apprenants du français vis-à-vis de cette langue et l'impact sur son enseignement-apprentissage, nous avons opté pour l'utilisation de la méthode d'investigation la plus répandue pour l'enquête sur le terrain, à savoir le questionnaire- (Javeau, 1982) -débat.

Le questionnaire-débat, analyse quantitative et qualitative, présente l'avantage de travailler sur des situations concrètes où le phénomène linguistique et culturel apparaît dans le même temps au niveau individuel et au niveau du groupe. En effet, il est l'outil le plus approprié et le plus pratique car spontané pour la collecte d'un grand nombre de données linguistiques. Il est nécessaire de rappeler que c'est une technique d'interrogation individuelle et de groupe, ce qui implique des réponses très intéressantes. De par son côté débat, il permet de dépasser les généralités ou de revenir sur les mêmes discours. Enfin, le questionnaire-débat autorise la confrontation des idées dans toutes leurs divergences et permet l'appréhension et la compréhension de l'autre.

\section{Déroulement de l'enquête}

Nous avons mené notre enquête, durant le mois de septembre 2013, au lycée public de la nouvelle ville de Tizi Ouzou, choisi pour des fins de proximité et de commodité de collecte des données.

Cette enquête a pour but principal de faire émerger les représentations à l'égard du français, et saisir l'impact de ces représentations sur l'enseignement-apprentissage de cette langue.

L'étude est effectuée en milieu scolaire et les informateurs visés par l'enquête sont représentés par un groupe composé d'une trentaine de lycéens dont le statut social est moyen et la situation professionnelle des parents hétérogène.

Le choix du palier «lycée » est justifié par le fait que l'élève, principal acteur de l'apprentissage, est censé avoir une connaissance solide de «l'outil » de l'apprentissage, celui qui lui permet d'aborder des études universitaires; l'accès aux études supérieures à Tizi Ouzou étant conditionné par le niveau de français car la plupart des filières (médecine, biologie, architecture, économie, gestion, langues) assurent leur enseignement en français.

Enfin, le lycéen est mieux placé que l'adolescent pour émettre ses opinions et ses représentations.

Les lycéens sont invités à répondre à ce genre de questions :

- Que représente le français pour vous?

- Que représentent pour vous la France et les Français?

- Les Français et les Algériens ont-ils des choses en commun ?

En répondant aux questions, les enquêtés ont manifesté leurs attitudes et représentations vis-à-vis de la langue française et du degré d'usage de cette langue dans la vie quotidienne. Les données orales recueillies ont été enregistrées et traitées dans l'analyse. 


\section{Analyse et interprétation succincte des réponses}

Les élèves étaient très motivés par le débat. Ils se sont laissé aller très spontanément au grès de leurs réflexions d'autant plus que l'enregistrement de leurs propos était mené de façon très discrète.

Pour eux, la France est tout d'abord un beau pays à visiter, dans lequel ils se projettent d'y faire des études, et/ou même d'y résider. C'est bien entendu le pays du savoir et de la science. Les Français sont beaux, travailleurs, méticuleux, toujours pressés, directs, ouverts et sérieux. Ils ont également un savoirvivre. Ce sont des gens qui se respectent et ne se mêlent pas de la vie des autres.

C'est aussi un peuple colonisateur et qui fait la guerre dans les pays musulmans.

Paradoxalement, d'autres disent que les Français sont racistes, n'aiment pas notamment les émigrés, les Arabes et les Noirs. Pour cette catégorie de personnes interrogées, adopter la langue des Français risque de provoquer la perte de l'identité arabo-musulmane et les Algériens qui s'expriment en français présentent des problèmes identitaires et psychologiques.

Ces images reçues proviennent principalement des chaines de télévision qui est une grande fenêtre ouverte sur le Monde, de l'école et des émigrés travaillant en France et rentrant régulièrement dans leur pays d'origine.

Il est nécessaire de souligner que l'environnement socioculturel de l'élève détermine et oriente ses représentations et son attitude linguistiques, le prédispose à "adhérer » aux modèles culturel et linguistique français, ce qui favorise la réussite dans l'apprentissage de la langue française. Nous devons également rappeler que la place qu'occupe le français, dans le système scolaire, par rapport aux autres disciplines, ne constitue pas un élément favorable à son apprentissage (cf. «Le français dans le système scolaire algérien » supra).

Pour la relation existant entre les Algériens et les Français, les réponses sont scindées en deux catégories : - la première basée sur ce qu'ils ont de commun : la ressemblance du climat, la Méditerranée qui les relie, la langue française commune ;

- l'autre axée sur les différences : la religion, les lieux et moyens de distraction, de loisirs.

\section{Questionnaire-débat : interprétation}

Les représentations sont sujettes à des variations; celles-ci peuvent avoir plusieurs origines : le sexe, le niveau socioprofessionnel des parents, la relation enseignant-enseigné, les difficultés relatives à la langue française, la pratique des langues locales et le milieu socioculturel dans lequel évoluent les apprenants. Nous allons montrer comment et pourquoi certaines "variables " permettent d'expliquer le désir et la motivation d'apprendre les langues et par la même la réussite ou l'échec de cet apprentissage.

Nous tenons également à préciser que nous nous situons dans le cadre d'une réflexion qui met en avant des interrogations sur le rapport de la langue française à la société algérienne en général et kabyle, en particulier.

A la question :

- « Aimez-vous le cours de français ? », nous avons constaté que les réponses sont divergentes. En effet, plusieurs variables ont un impact sur la motivation à l'apprentissage du français. Nous avons relevé les plus récurrentes. 


\subsection{La variable « sexe »}

Presque toutes les filles du groupe, en l'occurrence 25 sur 30 (83\%) ont répondu de manière spontanée et montrent leur intérêt pour cette langue plus que les garçons qui ne sont pas aussi motivés.

De manière générale, les filles sont portées sur les langues, surtout les langues étrangères qui, disent-elles, symbolisent la modernité, l'ouverture et le savoir. Les garçons quant à eux, sont plus attirés par les mathématiques, les sciences et les matières techniques.

Une lycéenne que nous appellerons Kamélia intervient pour dire que le cliché, aux filles les langues, et aux garçons les mathématiques et la technologie, est aujourd'hui complètement dépassé. En revanche, poursuit-elle, les filles obtiennent toujours et encore les meilleures notes en français et communiquent plus aisément dans cette langue.

- «Les filles aiment parler en français. C'est une marque de féminité pour elles...»

- «D'ailleurs, elles ne prononcent pas le «r $\mathrm{r}$ comme nous, les garçons, elles ne le roulent pas. C'est typique aux filles. »

En effet, ici, la langue n'offre pas l'image d'un moyen de communication qui remplit des fonctions sociales. Elle est considérée comme un critère de catégorisation. Celui ou celle qui ne communique qu'en français est un partisan de la France, un évolué ou encore un « civilisé », disent certains garçons.

\subsection{La variable « enseignant »}

Dans certaines interventions, les auteurs, une vingtaine sur trente $(66 \%)$ avancent un autre critère qui serait à l'origine de la présence ou l'absence de motivation pour l'apprentissage du français, à savoir que des paramètres relatifs à l'enseignant comme la méthode et la démarche de travail, la sympathie (ou l'antipathie), le "sérieux », peuvent déclencher ou freiner allant jusqu'à faire détester, chez l'élève, l'envie d'apprendre. Les commentaires entrant en considération:

- « J'aime le cours de français parce que le prof est gentil»

- «Il nous explique très bien les cours »

- « Il fait participer tous les élèves»

- « Notre enseignante nous comprend très bien »

Pour ceux qui n'apprécient pas ce cours de langue, leurs commentaires ressemblent à ceci :

- «Le prof de cette année ne répond jamais à nos questions »

- «Il ne nous fait que des exercices»

- « C'est tout le temps le chahut en classe, je déteste !»

- « Je regrette notre belle enseignante, jeune et toujours bien habillée »

\subsection{La variable « difficultés de la langue »}

Concernant le discours des élèves sur leur apprentissage du français, certains $(1 / 3$ des personnes interrogées) évoquent la difficulté de la langue française. Ces mêmes élèves, notamment ceux venus de régions arabophones, comparent l'apprentissage du français à celui de l'arabe. En effet, les lettres muettes, généralement prononcées par les apprenants du FLE, l'irrégularité des verbes présentant un obstacle dans la morphologie des verbes, la place de l'adjectif par rapport au terme qu'il qualifie, sont, entre autres, des difficultés qui démotivent les élèves et entravent l'apprentissage du français. En revanche, en langue arabe, tout ce qui s'écrit se prononce, il n'y a que trois voyelles et trois temps pour conjuguer les verbes.

- «L'arabe est plus facile à apprendre. Un simple exemple, en conjugaison, cette langue a seulement trois temps »

- « Dans notre langue arabe, tout est clair, il n'y a pas d'exceptions dans les règles grammaticales »

- «Toutes les matières sont enseignées en arabe, on connait bien cette langue à laquelle on est habitué »

Certains citent et précisent même l'aspect relatif à leurs difficultés. Les discours évoquent l'utilisation de la langue : 
- « Je ne peux pas parler le français en dehors des cours. J'ai beaucoup de difficultés et j'ai en même temps honte de parler cette langue parce que j'ai toujours peur de faire des fautes et de dire n'importe quoi »

- «Le français ne se prononce pas comme l'arabe, je n'aime pas lire à haute voix ... J'articule mal les lettres, c'est trop compliqué par rapport à l'arabe »

Pour d'autres, c'est la lecture et la compréhension :

- «Moi, je viens du sud de l'Algérie. Là-bas, on n'enseigne pas le français et quand mes parents sont arrivés à Tizi Ouzou, je devais suivre des cours en 2e année au collège.

Quand l'enseignante me demandait de lire, j'en étais incapable! Tout le monde se moquait de moi, tous les élèves riaient à chaque mot que j'essayais de prononcer... J'en ai souffert ! »

- « Je ne peux pas faire deux choses à la fois : lire et comprendre, soit je me concentre sur la lecture soit sur la compréhension »

Un élève dira que pour lui, la difficulté qu'il rencontre est l'apprentissage des règles.

- «Le français est une drôle de langue. On t'apprend une règle. Au moment où tu la retiens et que tu l'appliques on te dit que c'est faux : il y a des exceptions. Je ne comprends rien ! »

Un autre qui se présente arabophone déclare rencontrer beaucoup de difficultés dont la première se situe au niveau de l'oral.

- « Je confonds toutes les voyelles parce qu'il y en a tellement. Tout ce qui n'est pas « a », « ou», «i », je confonds. »

La plupart des élèves ont de réels problèmes au niveau de la prononciation de certains phonèmes, ce qui déforme les sons et fait construire des mots autres que ceux attendus.

Essayez de faire répéter le phonème [y], dans « prune » à un arabophone, il en est incapable, il produira un $[\mathrm{u}]$ ou [oe]. Cette confusion provient du fait que le $[y]$ est inconnu du locuteur car il n'existe pas dans sa langue maternelle.

Pour la plupart des élèves, la langue française est qualifiée de «compliquée » et même «très dure à apprendre ». Ce genre d'élèves ne fournissent aucun effort pour apprendre et améliorer leur niveau. En outre, ces mêmes élèves soutiennent que les mauvaises notes en français ne les empêchent pas de passer à la classe supérieure.

Seuls quelques élèves, notamment les filles, jugent que le français est une belle langue, facile à maitriser. Elle est associée à des stéréotypes valorisants :

- « J'aime beaucoup cette langue. J'attends le cours de français avec impatience »

- « C'est une belle langue»

- « Le français, j’adore !»

- «La séance de français nous permet de respirer, de voyager, de rêver !! »

- « C'est grâce au français qu'on a un bon avenir professionnel »

- « J'irai faire des études en France »

- « Je travaille le français parce que je veux vivre à l'étranger»

- «A l'université, les branches scientifiques et techniques (médecine, biologie, architecture, informatique) sont assurées en français, c'est pourquoi il faut avoir un bon niveau dans cette langue »

Les lycéens algériens ne se contentent pas seulement de livrer les représentations qu'ils se font du français, ils parlent aussi du peuple français. Ils voient chez les Français et en France des qualités qu'ils aimeraient avoir et voir dans leur pays. Par exemple, en France, les gens sont en sécurité ; la police contrôle tout ; la propreté est partout dans les hôpitaux, les rues...

- «En France, on n'a pas besoin de connaitre quelqu'un à la mairie pour se faire délivrer un acte d'état civil»

- «En France, avec ton argent tu peux tout acheter, il n’y a pas de pénurie dans les magasins »

- « En France, il y a des jardins entretenus, des espaces propres et bien fleuris »

- « Les Français respectent l'environnement... leurs cages d'escalier ne sont pas détruites et sales» 
Des propos qui mettent en valeur la supériorité de l'étranger (les Français) dans l'imaginaire des lycéens. S'agissant de lycéens kabyles, il paraitra normal pour certains qu'ils fassent l'éloge des Français : les Kabyles sont considérés comme des pro-français.

Certains avouent même être faibles en français, ils déclarent avoir de grandes lacunes. Ils accusent les enseignants et les programmes d'être responsables de leur faible niveau. En revanche, ils veulent apprendre le français et espèrent le parler comme l'arabe.

- «Avant, je ne savais pas que les langues sont importantes. Aujourd'hui, je veux rattraper le retard en français... Je suis des cours privés »

- «Moi, je veux parler les langues, le français, l'arabe et l'anglais »

- «Pour voyager, il faut connaitre les langues, c'est important»

- « Vous voyez les Chinois... il y en a beaucoup à Tizi Ouzou et ils parlent Kabyle... qui pourrait le dire il y a quelques années en arrière? »

\subsection{La variable « Le français, langue de la colonisation »}

L'image de la langue française comme langue de la colonisation est la représentation de ceux qui sont hostiles à l'apprentissage du français. Ces lycéens évoquent une période passée où la langue française était rejetée par leurs parents parce qu'elle représentait la langue du colonisateur, la langue de l'ennemi. Pour cette catégorie d'élèves (une minorité) qui, précisent-ils, s'absentent aux cours très souvent, le français est inutile et n'a aucun impact ni sur leur réussite scolaire ni sur leur vie professionnelle.

La langue française est dévaluée par les discours politique et religieux anti-français et pro-arabes jouant sur le nationalisme de la population et rappelant en permanence que l'arabe est la langue du coran, la langue de la parole divine; ce qui démontre le rejet du français et l'image négative à inculquer aux élèves.

La société algérienne est une société de plus en plus marquée par la religion ; de ce fait, les concepteurs algériens des manuels scolaires repoussent et excluent tout ce qui n'en fait pas partie. En effet, si la culture occidentale libère l'individu, ce n'est pas le cas de la culture arabe.

L'occupation et la colonisation françaises toujours présentes dans l'esprit « nationaliste » ne facilitent pas la valorisation de la culture française et inhibent son introduction dans les manuels scolaires.

\subsection{La variable « catégorie socioprofessionnelle »}

Cette variable touche la situation socioprofessionnelle des parents. Le contact qu'ont les élèves de Tizi Ouzou avec la langue française ne se limite pas seulement à l'école, ce qui constitue un critère saillant dans leurs représentations de la langue française.

Le cas des parents fonctionnaires est annoncé par la plupart (70\%) des élèves; nous pouvons en déduire qu'ils proviennent de catégorie socioprofessionnelle moyenne. Les enfants de cadres supérieurs et de professions libérales fréquentent, quant à eux, l'école privée c'est-à-dire l'école française.

- «Les élèves qui entendent parler leurs parents en français à la maison, à leur tour, vont parler cette langue dès que l'occasion leur est offerte. Le français est également présent dans le milieu kabyle : la télé, l'Internet... ces élèves bien familiarisés avec le français sont prédisposés à l'apprendre plus encore »

- «Pour un élève dont les parents ne communiquent qu'en arabe le français est une langue étrangère dure à apprendre »

\subsection{La variable « plurilinguisme »}

Le plurilinguisme algérien est problématique car les langues maternelles ne sont pas enseignées (sauf la langue berbère, qui est enseignée seulement en Kabylie et ce, depuis 2002).

Nous avons demandé à nos interlocuteurs :

«Comment situez-vous le français par rapport aux autres langues en présence en Algérie ?» 
La question a suscité de vives réponses. Les jeunes voulaient tous prendre la parole en même temps. Tous étaient unanimes pour déclarer ces réalités :

- « Les habitants de Tizi Ouzou sont kabylophones de naissance. Ils communiquent en kabyle, en français et en arabe algérien. Cependant, des arabophones sont venus s'établir dans cette ville. Leurs enfants « baragouinent » le kabyle qu'ils comprennent, lorsqu'ils se retrouvent avec leurs pairs »

Dans cette variable «le plurilinguisme», nous allons situer la langue française par rapport aux autres langues en présence. Pour ce faire, des « qualifications » servent à situer et à classer les langues suivant le statut accordé par les politiques linguistiques : internationale, nationale, officielle... Des classifications qui relèvent des rapports de force. Par exemple, en Algérie, après l'indépendance en 1962, malgré le manque de moyens, les membres du gouvernement ont installé une politique linguistique d'arabisation pour affirmer l'identité arabe sans même penser à préserver les autres langues du peuple « L'arabisation est devenue synonyme de ressourcement, de retour à l'authenticité, de récupération de l'identité arabe qui ne peut se réaliser que par la restauration de la langue arabe, récupération de la dignité bafouée par les colonisateurs ». (Taleb Ibrahimi, 1997:184).

Pour ce qui est des langues parlées, les réponses les plus fréquentes concernent la cohabitation : arabe algérien - français ; arabe algérien - kabyle ; kabyle - français ; arabe algérien - kabyle - français.

Ce mélange de langues dans les communications quotidiennes et spontanées indique un plurilinguisme spécifique au milieu algérien. La langue française cohabite en harmonie avec le kabyle mais également avec l'arabe.

Le plurilinguisme est parfois associé à l'oubli, à un mélange. Les enquêtés ont répondu ainsi :

- «En cours de français, quand ils sont interrogés, certains copains " cherchent» leurs mots. Quand ils doivent répondre aux questions, ils s'arrêtent à chaque mot, ils doivent réfléchir, et après, s'ils ne le trouvent pas en français ils le prennent du kabyle ou de l'arabe »

Un élève insiste :

- «Le mélange de langues est très courant chez nous, les Algériens »

La compétence communicative des élèves est approximative dans toutes les langues en présence, c'est pourquoi ils recourent toujours à un mélange de langues.

La langue française est utilisée au quotidien par les Algériens, en général, par de nombreux lycéens, en particulier, aussi bien dans les domaines formel qu'informel. Ainsi la grande majorité des enquêtés affirment communiquer en français, essentiellement en classe et pour lire les journaux, revues et bien entendu, surfer sur Internet.

Pour les uns, c'est parce qu'ils y sont obligés, pour d'autres parce qu'ils aiment et apprécient cette langue. - «On a besoin du français pour suivre les programmes de télévision sur les chaines françaises »

- « Pour envoyer des SMS à mes copains, j’utilise le français, j’ai pas le choix »

Le taux d'utilisation du français par les filles est bien supérieur à celui des garçons.

L'intérêt pour la lecture constaté dans les réponses des filles dépasse celui des garçons. En effet, les réponses des enquêtées révèlent une attirance pour la lecture en langue française.

Les lycéens sont tous conscients et du même avis sur la nécessité de l'apprentissage des langues en général, du français plus particulièrement, connaissant toutes les opportunités que cette langue peut leur offrir.

Rares sont les jeunes qui pensent qu'il faut privilégier la langue arabe standard et éviter les émissions françaises pouvant porter atteinte aux traditions et aux croyances algériennes.

Cette importance qu'ils lui accordent est une représentation qui les motive à fournir des efforts pour parvenir à de bons résultats et une bonne pratique langagière.

Pour mieux appréhender la question de l'importance de la langue française dans l'enseignement, nous avons vérifié la question en corrélation avec les niveaux d'étude au lycée.

A la question:

«Pensez vous que l'arabisation des matières scientifiques est un handicap pour maitriser la langue française ?» 
La grande majorité des jeunes élèves (95\%) ont répondu que l'arabisation est la cause principale de l'échec scolaire et de la dévalorisation du français. Ils ne cachent pas non plus leurs angoisses surtout par rapport à leur cursus universitaire où les filières les plus importantes et les plus intéressantes sont dispensées en français notamment à l'université de Tizi Ouzou.

A la dernière question :

" Comment voyez-vous l'avenir de la langue française en Algérie? »

Les jeunes lycéens déclarent qu'il est impossible que cette langue soit concurrencée par d'autres parce qu'elle n'est pas une langue étrangère mais fait partie du quotidien de chacun.

- «Le français n'a jamais été et ne sera jamais une langue étrangère aux Kabyles même si le gouvernement soutient le contraire»

- « Je ne peux pas imaginer l’Algérie sans le français ».

\section{Synthèse}

L'enquête menée nous a permis de constater qu'en général, la langue française a un statut privilégié dans les représentations des jeunes lycéens de la ville de Tizi Ouzou. Une langue, disent-ils, qui permet l'ouverture de l'esprit et l'ouverture sur le monde ; une langue qui véhicule le savoir, la modernité et même qui affiche leur identité ! Ils regrettent, cependant, qu'elle ne soit plus la langue d'enseignement des matières scientifiques et techniques dans le système éducatif comme cela était juste après l'indépendance.

L'enquête montre bien l'attachement des apprenants kabylophones à la langue française, ce qui favorise la pratique aisée et aide à son apprentissage.

Parallèlement, cette même langue reçoit un autre type de représentations : c'est une langue trop difficile, avec une grammaire trop compliquée ; c'est la langue du colonisateur, de l'ennemi, des considérations qui peuvent ralentir le processus d'apprentissage jusqu'à même le bloquer et pousser vers l'échec. Un échec pouvant également provenir des contenus des programmes scolaires non-adaptés non seulement au niveau des élèves mais aussi à leurs besoins et exigences.

Au terme de cet article, nous nous apercevons de l'importance de la prise en compte des représentations des apprenants, dans le système éducatif. En effet, en vue de l'élaboration des stratégies d'enseignementapprentissage appropriées aux élèves, il est non seulement nécessaire de s'intéresser à leur environnement social, à leurs besoins mais aussi à leurs attitudes et représentations face aux langues.

Les résultats et suggestions proposés dans cet article sont loin de prétendre à l'exhaustivité, ils gagneraient à être complétés par des recherches ultérieures. En effet, ce travail a permis de cerner des éléments de la représentation du français chez certains apprenants de la ville de Tizi Ouzou, cependant, il pourrait être enrichi par d'autres enquêtes apportées, par exemple, par les enseignants et les parents d'élèves.

\section{Références bibliographiques}

Auger, N. (2003). « Manuels et stéréotypes ». Le français dans le monde n 326, Paris : Clé International.

Beacco, J-C. (1995). Les dimensions culturelles des enseignements de langue. Paris : Hachette.

Canut, C. (2000). "Subjectivité, imaginaires et fantasmes des langues : la mise en discours épilinguistique ». Langage et société, $\mathrm{n}^{\circ}$ 93, pp.71-98.

Benrabah, M. (1999). Langue et pouvoir en Algérie, Paris : Seguier.

Castelloti, V. et Moore D. (2002). Représentations sociales des langues et enseignements. Guide pour l'élaboration de politiques linguistiques éducatives en Europe. De la diversité linguistique à l'éducation plurilingue. Strasbourg : Conseil de l'Europe. 
Chaker, S. (1989). Berbères aujourd'hui. Paris : L'Harmattan.

Cheriguen, F. (1997). « Politique linguistique en Algérie ». Mots, les langages du politique, n 52, pp. 62-73.

Collès, L. (1992). « Des représentations aux productions en langue étrangère dans le cadre scolaire ». Repères 6, pp. $23-40$.

Dabene L. (1997) «L'image des langues et leur apprentissage ». Matthey, M. Les langues et leurs images, pp.19-23.

Grandguillaume, G. (2004). « La Francophonie en Algérie ». Hermès $n^{\circ}$ 40, pp. 75-78.

Grandguillaume, G. (1997). «L'oralité comme dévalorisation linguistique ». Peuples Méditerranées, Langues et stigmatisation sociale au Maghreb, $\mathrm{n}^{\mathrm{o}} 79$, pp. 9-15.

Grandguillaume, G. (1983). Arabisation et politique linguistique au Maghreb. Paris : Maisonneuve et Larose.

Javeau, C. (1982). L'enquête par questionnaire. Université de Bruxelles.

Moore, D. (2003). Plurilinguisme et école, représentations et dynamisme d'apprentissage, synthèse de l'activité de recherche. Université Stendhal Grenoble III.

Moore, D. et al. (2001). Les représentations des langues et de leur apprentissage : références, modèles, données et méthodes. Paris : Didier.

Morsly, D. (1988). Le français dans la réalité algérienne. Thèse de doctorat d'État, Université de Paris V.

Muller, N. (1998). «L'allemand, c'est pas du français! » Enjeux et paradoxes de l'apprentissage de l'allemand, Neuchâtel : INRP-LEP.

Outaleb-Pellé, A. (2013). «Pratiques et représentations du français au sein de deux familles kabyles de Tizi Ouzou ». Sini. Ch. Les langues dans l'espace familial algérien, Oran, Crasc, pp. 95-109.

Outaleb-Pellé, A. (2013). «L'enseignement du FLE : réflexion sur la culture ». (Arezki, D. dir.). Enseignement et apprentissage des langues étrangères en Algérie, université de Tizi Ouzou, pp. 37-51.

Outaleb-Pellé, A. (2010). Erreurs d'apprenants du FLE. Le cas des lycéens de Tizi Ouzou. Thèse de doctorat, université Nice-Sophia Antipolis.

Porcher, L. (2004). L'enseignement des langues étrangères. Paris : Hachette.

Py, B. (2000). Représentations sociales et discours. Questions épistémologiques et méthodologiques, Tranel $\mathrm{n}^{\circ} 32$, pp. 5-20.

Taleb-Ibrahimi, K. (1995). Les Algériens et leur(s) langue(s). Alger : El Hikma.

Temim, D. (2007). Nomination et représentation des langues en Algérie. (Cheriguen, F. dir.). Enjeux de la nomination des langues dans l'Algérie contemporaine. Paris, L'Harmattan, pp. 19-35.

Véronique, D. (1990). «A la rencontre de l'autre langue : réflexions sur les représentations dans l'apprentissage d'une langue étrangère ». Le Français dans le Monde, Recherches et Applications.

Zarate, G. (1993). Représentations de l'étranger et didactique des langues. Paris : Didier. 